\title{
Comparison of GeneXpert MRSA/SA ETA assay with semi-quantitative and quantitative cultures and nuc gene-based qPCR for detection of Staphylococcus aureus in endotracheal aspirate samples
}

Jasmine Coppens ${ }^{1}$, Liesbet Van Heirstraeten ${ }^{1}$, Alexey Ruzin², Li Yư², Leen Timbermont ${ }^{1}$, Christine Lammens ${ }^{1}$, Veerle Matheeussen ${ }^{3}$, Michael McCarthy ${ }^{2}$, Philippe Jorens ${ }^{4}$, Margareta leven ${ }^{1,3}$, Samir Kumar-Singh ${ }^{1,5}$, Herman Goossens ${ }^{1,3}$ and Surbhi Malhotra-Kumar ${ }^{1 *}$ (D)

\begin{abstract}
Introduction: Staphylococcus aureus (S. aureus) is a common cause of ventilator-associated pneumonia. Rapid and accurate detection of lower respiratory tract colonization and/or infection with S. aureus may inform targeted preventive and therapeutic strategies. To investigate this, we compared semi-quantitative (SQ)-culture results from 79 endotracheal aspirates (ETA) collected from mechanically-ventilated patients, to two culture and two non-culture-based methods for detection of S. aureus.

Methods: ETA analyzed by routine SQ-culture on blood and colistin-nalidixic-acid agar was compared to: (i) quantitative (Q-) culture on chromogenic COLOREX ${ }^{\mathrm{TM}}$ Staph aureus; (ii) enrichment in brain-heart-infusion broth followed by plating on blood agar and COLOREX'"; (iii) nuc-based TaqMan qPCR, and (iv) GeneXpert MRSASSA ETA assay.

Results: Of the 79 ETA samples analyzed by SQ-culture, 39 samples were positive, and 40 negative for $S$. aureus. Two samples negative for S. aureus by SQ-culture were, however, S. aureus-positive by the other four methods and were considered positive. Appending these two samples as positive in the SQ-culture results, sensitivities-specificities for Qculture, enrichment-culture, TaqMan qPCR and GeneXpert were 100-95, 100-92, 100-53\% and 100\%-100, respectively. The lower specificities of Q-culture, enrichment-culture, and TagMan QPCR was because of their higher sensitivities, although TaqMan qPCR also detected S. aureus-specific extracellular DNA.

Conclusion: This first evaluation of the GeneXpert MRSA/SA ETA assay with ETA samples found it to be highly sensitive, specific, user-friendly (hands-on time $\sim 5 \mathrm{~min}$.), and rapid ( $66 \mathrm{~min}$. assay time). Where this equipment is not available, we recommend implementing more sensitive culture-based methods for improved S. aureus detection in ETA samples.
\end{abstract}

Keywords: Cepheid, Real-time PCR, Rapid diagnostics, VAP, Ventilator-associated pneumonia, COLOREX'M staph aureus, Chromogenic medium

\footnotetext{
* Correspondence: surbhi.malhotra@uantwerpen.be

${ }^{1}$ Laboratory of Medical Microbiology, Vaccine \& Infectious Disease Institute,

University of Antwerp, Universiteitsplein 1, D.S.6.23, 2610 Wilrijk, Belgium

Full list of author information is available at the end of the article
}

(c) The Author(s). 2019 Open Access This article is distributed under the terms of the Creative Commons Attribution 4.0 International License (http://creativecommons.org/licenses/by/4.0/), which permits unrestricted use, distribution, and reproduction in any medium, provided you give appropriate credit to the original author(s) and the source, provide a link to the Creative Commons license, and indicate if changes were made. The Creative Commons Public Domain Dedication waiver (http://creativecommons.org/publicdomain/zero/1.0/) applies to the data made available in this article, unless otherwise stated. 


\section{Introduction}

Methicillin-sensitive and -resistant Staphylococcus aureus (MSSA and MRSA) cause life-threatening infections in both high-risk and in healthy individuals [1]. One of the most common nosocomial infections where $S$. aureus is an important causative agent is ventilator-associated pneumonia (VAP) [2]. VAP not only results in a substantial increase in morbidity and mortality but also in a costly prolongation of patient bed days. The classical definition of VAP is based on clinical signs, a new infiltrate on the chest X-ray and, importantly, the detection of the causative pathogen from respiratory samples $[3,4]$.

Prior colonization with potential pathogens plays a significant role in the development of nosocomial infections [5]. For instance, intensive care unit (ICU) patients with $S$. aureus colonization have up to a 15-fold higher risk of developing VAP compared to non-colonized patients [6]. These data suggest that pathogen identification by surveillance culture could represent a preemptive strategy for VAP. ETAs have the advantage of being a noninvasive sample that can be obtained rapidly and repeatedly with fewer complications and resources, and are therefore commonly used for routine microbiological surveillance cultures [7].

While culture of pathogen remains the gold standard, molecular tests that typically have a shorter turnaround time can drastically decrease the critical time-to-initiation of preventive and therapeutic strategies, including the initiation of the appropriate antibacterial treatment. A recently introduced molecular test, currently utilized for research purposes only, that directly detects $S$. aureus from ETA samples is the GeneXpert SA/MRSA ETA assay. Here, we evaluated and compared the GeneXpert assay to culture and non-culture based methods for detection of $S$. aureus in ETA samples.

\section{Materials and methods \\ Study design and sample collection}

The study was designed to assess the performance of a rapid screening test, the GeneXpert MRSA/SA ETA assay, in patients at risk of developing VAP. During May 2017February 2018, ETA samples were collected from mechanically ventilated adult patients at the ICU of the University Hospital of Antwerp, either as surveillance cultures or as routine samples obtained in patients with suspicion of (pulmonary) infection. ETAs were analysed immediately at the Clinical Microbiology laboratory of the University Hospital of Antwerp using quadrant-based, semi-quantitative (SQ)-culture on blood agar, chocolate agar, McConkey and colistin-nalidixic acid agar (CNA, Oxoid) and bacterial growth was evaluated after $24 \mathrm{~h}$ of incubation [8]. Based on quadrant growth, SQ-culture method categorizes positive samples as light (growth in quadrant 1), moderate (growth in quadrants 2 and also 3 ) and heavy (growth in all four quadrants). For every $S$. aureus-positive sample studied, a negative sample was also included. At the end of study period, 79 samples were collected as $S$. aureus negative ( $n$ $=40)$, light $(n=19)$, moderate $(n=10)$ and heavy $(n=10)$ (Additional file 1: Table S1). SQ-culture was followed by disk diffusion with cefoxitin $(4 \mu \mathrm{g} / \mathrm{ml})$. The samples were further processed within $48 \mathrm{~h}$ after ETA collection with different methods for $S$. aureus detection and quantitation at the Laboratory of Medical Microbiology, University of Antwerp. Methods used were: GeneXpert MRSA/SA ETA Assay; quantitative (Q-) culture on chromogenic COLOREX $^{\mathrm{TM}}$ Staph aureus; enrichment broth followed by blood agar and COLOREX plating; and TaqMan qPCR targeting the single-copy thermostable nuclease (nuc) gene on extracted DNA. In case needed, the samples were stored at $4{ }^{\circ} \mathrm{C}$ until further processing (maximum $48 \mathrm{~h}$ ). The study was approved by the ethics committee of the Antwerp University Hospital (Belgian registration number B300 201629199).

\section{GeneXpert assay}

The GeneXpert assay is currently available for research (RMRSA/SA-ETA-10, Cepheid, USA) and was performed according to manufacturer's instructions. Briefly, ETA samples were adsorbed onto a Cepheid collection device swab (COPAN- 900-0370, USA), dissolved in elution buffer and vortexed at high speed for $10 \mathrm{~s}$ (Scientific Industries Inc., USA). The entire content of the elution reagent was transferred to the cartridge and analysed by GeneXpert ${ }^{\circledR}$ Dx system v4.7b (Cepheid, USA). The overall process of extraction, amplification, and detection of the targets was completed in $66 \mathrm{~min}$. The primers and probes in the MRSA/SA ETA assay detect sequences within the staphylococcal protein A $(s p a)$ gene, the methicillin resistance gene (mecA), and the staphylococcal cassette chromosome (SCCmec) inserted into the $S$. aureus chromosomal $a t t B$ insertion site [9]. Samples were reported by GeneXpert as either $S$. aureus "detected" (Ct 3-36) or "not detected".

\section{Sample preparation for comparator culture-based and qPCR methods}

After initiating the GeneXpert assay, the remaining ETA sample was homogenised by blending and liquefaction with $\mathrm{N}$-acetylcysteine. All samples were blended with the dispersing instrument T10 basic ULTRA-TURRAX (IKA, Staufen, Germany) for $10 \mathrm{~s}$ at maximum speed on ice. Depending on the viscosity of the sample, checked by visual inspection, the blending time was increased by steps of $20 \mathrm{~s}$, with a maximum blending time of $60 \mathrm{~s}$. After blending, the samples were liquefied with lysomucil (10\% N-acetylcysteine, Zambon, Milan, Italy). $3 \mathrm{ml}$ lysomucil was dissolved in $12 \mathrm{ml}$ phosphate buffered saline (PBS), and an equal amount in volume $(\sim 300 \mu \mathrm{L})$ of liquefying reagent was added as $1: 1$ to the sample, and vortexed at full speed for $10 \mathrm{~s}$. After incubation at $37^{\circ} \mathrm{C}$ 
for $15 \mathrm{~min}$, the samples were vortexed again at full speed for $10 \mathrm{~s}$, and the incubation step was repeated.

\section{Quantitative cultures}

Serial dilutions of the liquefied samples were spiral-plated (Eddy Jet, program 6; $50 \mu \mathrm{L}$ logarithmic spreading; IUL, Spain) on the chromogenic COLOREX ${ }^{\mathrm{Tx}}$ Staph aureus medium (bioTRADING, The Netherlands) and on blood agar (Columbia II Agar Base, Oxoid, UK with 5\% defibrinated horse blood). After $24 \mathrm{~h}$ of incubation, pink to mauve $S$. aureus colonies on the COLOREX ${ }^{m}$ Staph aureus were counted and S. aureus loads were calculated as colony forming units $(\mathrm{CFU}) / \mathrm{ml}$ for each sample. At least one pink to mauve colony per plate was speciated by MALDI-TOF (Bruker, USA) and stored at $-80^{\circ} \mathrm{C}$. Q-culture results were also correlated with VAP incidence in 13 patients when the VAP diagnosis according to the classical definition had been made on the day \pm 1 of sample collection $[3,4]$.

\section{Enrichment-based cultures}

Additional enrichment was performed by overnight incubation of a small volume $(\sim 100 \mu \mathrm{l})$ of the liquefied sample in brain heart infusion (BHI) broth followed by plating on COLOREX $^{\mathrm{m}}$ Staph aureus as well as on blood agar plates. Presumptive $S$. aureus colonies were confirmed by MALDI-TOF (Bruker, USA).

\section{nuc gene-based qRT-PCR}

$200 \mu \mathrm{L}$ of the liquefied sample was subjected to proteinase $\mathrm{K}$ treatment for $15 \mathrm{~min}$ at $56^{\circ} \mathrm{C}$ followed by automated DNA extraction (NucliSENS ${ }^{\bullet}$ EasyMag ${ }^{\circ}$, bioMérieux SA, France) and frozen until batched analysis by the nuc gene-based qPCR assay was performed. Concentrations of S. aureus DNA were determined using quantitative TaqMan real-time PCR targeting the single-copy nuc gene. qPCR was performed in a $20 \mu \mathrm{L}$ reaction volume containing 2x Taqman $^{\text {Tm }}$ Universal PCR Master Mix (Applied Biosystems $^{\mathrm{nN}}$, California, USA), $200 \mathrm{nM}$ concentrations of primers SAnucF2 (TAAAGCGATTGATGGTGATACG), SAnucR2 (TTCTTTGACCTTTGTCAAACTCG), TaqMan probe (cy5-TGGTCCTGAAGCAAGTGCATTTACg-BBQ) and $4 \mu \mathrm{L}$ of DNA template. Amplification was carried out on CFX96 Touch $^{\text {тm }}$ Real-Time PCR detection system (Bio-Rad Laboratories Inc., California, USA) using the following cycling parameters: $5 \mathrm{~min}$ at $95^{\circ} \mathrm{C}$ and 40 cycles of $10 \mathrm{~s}$ at $95^{\circ} \mathrm{C}$ and $50 \mathrm{~s}$ at $60^{\circ} \mathrm{C}$. Bacterial loads were calculated based on a standard curve that was set up using Avogadro's constant and the molecular weight of serially diluted nuc PCR product of SA NCTC 8325 [10]. Samples and standard curves were run in triplicate and samples giving $\mathrm{Ct}$ values $\leq 37$ were considered positive for $S$. aureus.

\section{Results}

GeneXpert MRSA/SA ETA assay is a rapid and accurate method for detection of $S$. aureus in ETA samples

Additional file 1: Table S1 gives an overview of the analysed results of the 79 ETA samples utilizing SQ-culture, GeneXpert assay, Q-culture, enrichment-culture and the nuc gene-based qPCR. All samples positive for SQ-culture (39/ 79) were also positive for each of the other four methods, resulting in 100 sensitivities with SQ-culture as gold standard/comparator. The specificities of the tests, however, differed. The GeneXpert assay directly detects $S$ aureus in unprocessed ETA samples based on detection of the spa gene by real-time amplification where a Ct value between 3 and 36 is deemed positive. With 41/79 samples positive for S. aureus, GeneXpert showed a $97.5 \%$ concordance with SQ-culture methods, discording for only two samples (samples 39 and 40) that were positive by GeneXpert (both with $\mathrm{Ct}$ values of 29) but negative by SQ-culture at $24 \mathrm{~h}$ (both samples grew S. aureus at $48 \mathrm{~h}$ ). However, these two samples were also positive by Q-culture, enrichment-based culture and by $n u c$ qPCR and were therefore assessed as positive, and along with SQ-culture results, comprised the positive samples in the extended gold standard panel (Additional file 1: Table S1). With the extended gold standard panel as a comparator, GeneXpert assay showed 100\% sensitivity and specificity (Table 1 ). Taken together with the short total assay time $(<70 \mathrm{~min})$ for GeneXpert, this assay was assessed as the best for direct detection of S. aureus in ETA samples in this comparator study.

Quantitative and enrichment cultures have slightly higher sensitivities than semi-quantitative culture

Next, we studied Q-cultures on the chromogenic COLOREX $\mathrm{X}^{\mathrm{sm}}$ Staph aureus medium that is also commonly utilized by clinical laboratories. The sensitivity and

Table 1 Sensitivities and specificities of the five methods utilized for S. aureus detection in ETA samples compared to the extended gold standard

\begin{tabular}{|c|c|c|c|c|c|}
\hline & & \multicolumn{2}{|c|}{ Extended gold standard* } & \multirow{2}{*}{$\begin{array}{l}\text { Sensitivity (\%) } \\
(95 \% \text { C1) }\end{array}$} & \multirow{2}{*}{$\begin{array}{l}\text { Specify (\%) } \\
(95 \% \text { C1) }\end{array}$} \\
\hline & & + & - & & \\
\hline \multirow[t]{2}{*}{ GeneXpert } & + & 41 & 0 & 100 & 100 \\
\hline & - & 0 & 38 & & \\
\hline \multirow[t]{2}{*}{ Q-culture } & + & 41 & 2 & 100 & 94.74 \\
\hline & - & 0 & 36 & & $(82.25-99.36)$ \\
\hline \multirow[t]{2}{*}{ Enrichment } & + & 41 & 3 & 100 & 92.11 \\
\hline & - & 0 & 35 & & (78.62-98.34) \\
\hline \multirow[t]{2}{*}{ qPCR } & + & 41 & 18 & 100 & 52.63 \\
\hline & - & 0 & 20 & & $(35.82-69.02)$ \\
\hline \multirow[t]{2}{*}{ SQ-culture } & + & 39 & 0 & 95.12 & 100 \\
\hline & - & 2 & 38 & $(83.47-99.40)$ & \\
\hline
\end{tabular}

*S. aureus detected by SQ-culture plus two samples that showed S. aureus presence by the other four methods but not by standard culture 
specificity of Q-culture, calculated against the extended gold standard panel were 100 and 95\%, respectively, with 43/79 ETA being positive for S. aureus (Table 1). Lower specificity of Q-culture was due to the fact that $S$. aureus was detected in two additional samples (samples 37 and 38 with $S$. aureus loads of 848 and $40 \mathrm{CFU} / \mathrm{ml}$, respectively) that were negative by both SQ-culture and GeneXpert but confirmed to be positive by enrichment-based culture and by qPCR.

Enrichment-based culture identified one additional ETA sample as positive for $S$. aureus that was only confirmed by in-house qPCR. Thus, with 44/79 samples being positive after enrichment, this method showed a $100 \%$ sensitivity and $92 \%$ specificity with the extended gold standard panel as a reference. Thus, the increased sensitivities of Q-culture and enrichment culture led to lower specificity compared to SQ-culture and to GeneXpert.

Furthermore, based on the SQ-culture results, 7 patients $(41 ; 43 ; 44 ; 50 ; 53 ; 58 ; 75)$ were diagnosed with VAP due to $S$. aureus (MSSA, $n=6$; MRSA $n=1$ ) on the day \pm 1 of sample collection. We studied whether the $S$. aureus loads by Q-culture on COLOREX ${ }^{\text {mo }}$ Staph aureus plate were higher in samples obtained from the $7 \mathrm{pa}$ tients who developed VAP due to $S$. aureus compared to S. aureus-positive patients not developing VAP. Interestingly, the $S$. aureus VAP group showed higher loads (median $1.6 \times 10^{6} \mathrm{CFU} / \mathrm{ml}$, range: $10^{1} \mathrm{CFU} / \mathrm{ml}-10^{8} \mathrm{CFU} /$ $\mathrm{ml}$ ) than the $S$. aureus VAP-negative group (median $6.4 \times 10^{2} \mathrm{CFU} / \mathrm{ml}$, range: $\left.10^{\circ} \mathrm{CFU} / \mathrm{ml}-10^{8} \mathrm{CFU} / \mathrm{ml}\right)$, although the differences in loads remained non-significant $(P=0.806)$ (Fig. 1). Also, the correlation of the Ct values of the GeneXpert and qPCR was investigated. In 5 of the 7 samples, the $\mathrm{Ct}$ value of qPCR was higher than the $\mathrm{Ct}$ of the GeneXpert and one sample each, the Ct of qPCR were lower and the same as GeneXpert $(P=0.09)$ (Additional file 1: Table S1).

\section{In-house qPCR was the most sensitive but also the least specific assay for $S$. aureus detection in ETA samples} We also developed a nuc gene-based qPCR whose analytical performance was extensively validated with $S$. aureus-negative ETAs spiked with different concentrations of $S$. aureus prior to utilization in this study (data not shown). The limit of detection of the qPCR in ETAs was $\sim 10^{3}$ $\mathrm{CFU} / \mathrm{ml}$ (equals 11.4 genome equivalents detected in the total DNA input in the PCR), and the upper limit of quantification was $>10^{6} \mathrm{CFU} / \mathrm{ml}$, and is in agreement with the diagnostic thresholds for ETA of $10^{5}-10^{6} \mathrm{CFU} / \mathrm{mL}$ [11]. With this assay, 59/79 (75\%) ETA samples were positive for S. aureus and with the extended gold standard panel as reference, showed a $100 \%$ sensitivity and $53 \%$ specificity (Table 1). Utilizing the enrichment-based culture method as a reference, that we believe was the most sensitive assay here, the sensitivity of nuc qPCR was $100 \%$, however, specificity was only marginally increased to $57 \%$. Thus, while all samples identified as S. aureus-positive by any of the other four methods were all detected as positive by the qPCR, an additional 15 ETA samples were also identified as positive by this method with Cts ranging from 30 to 37 .

\section{Discussion}

Early and reliable screening for $S$. aureus colonization or infection of the lower respiratory tract may inform targeted and novel preventive and therapeutic strategies. With this in mind, we studied and cross-compared 2 PCR-based and 3 culture-based methods for $S$. aureus detection in ETA samples, including a SQ-culture on blood and colistin-nalidixic-acid agar routinely used in

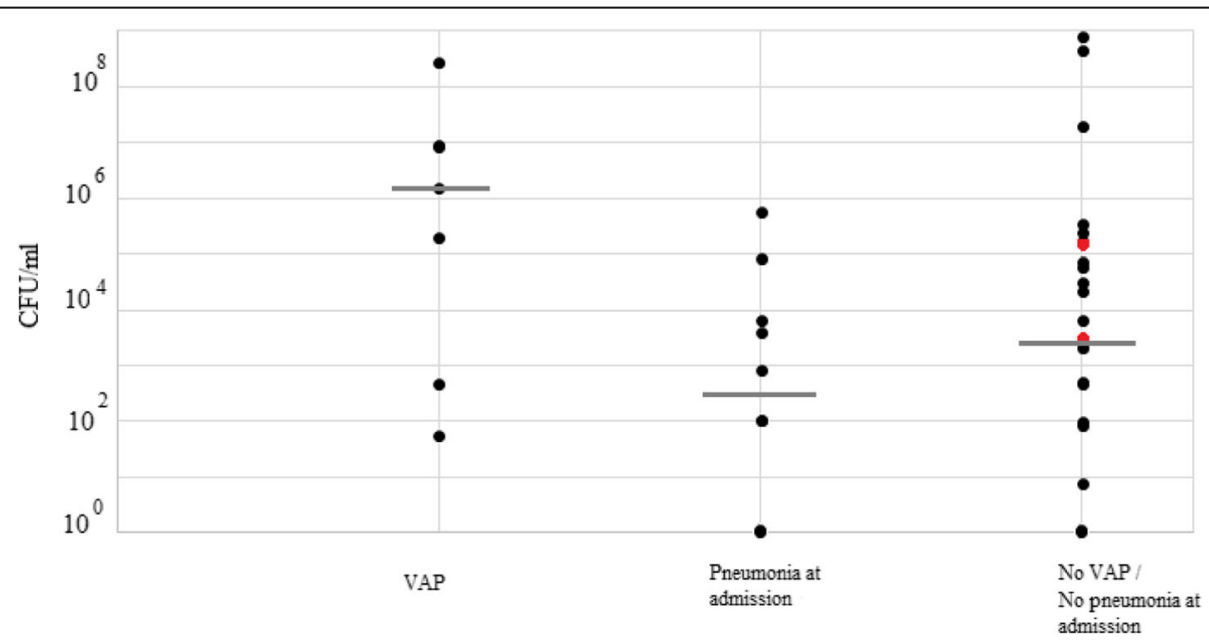

Fig. 1 S. aureus loads in ETA samples from patients diagnosed with VAP on the day of sample collection or not (no VAP) or having pneumonia at ICU admission. The two red dots represent two ETAs that showed S. aureus presence by the other four methods but not by SQ-culture. Gray lines represent the median 
our hospital. With the criteria of at least two methods simultaneously detecting $S$. aureus, enrichment-based culture and the in-house nuc-based qPCR emerged as the most sensitive methods for detection of $S$. aureus in ETA samples in this study. All 44 samples that showed the presence of $S$. aureus by enrichment-based culture were also positive by qPCR. However, of these, only 43 samples were positive by Q-culture, 41 by GeneXpert, and 39 by SQ-culture (Additional file 1: Table S1). The pre-treatment with lysomucil in the concentrations used was not inhibitory for $S$. aureus recovery here. The inhibitory role of $\mathrm{N}$-acetylcysteine (NAC) to bacterial growth was shown previously [12], but we were able to show that Q-culture (NAC treated) was more sensitive than SQ- and GeneXpert (non-NAC treated). Only one sample that was $S$. aureus positive by enrichment broth was found negative by Q-culture but this sample was also negative on SQ-culture and by GeneXpert with only the q-PCR showing a positive result with a high $\mathrm{Ct}$ value of 38. Enrichment-based cultures also offered the best proof of $S$. aureus identification as these samples were also validated with MALDI-TOF-based $S$. aureus confirmation. The described optimized protocols for selective and enrichment-based culture methods can be employed for more sensitive S. aureus detection. However, the time cost in culture-based methods will always be an issue for routine clinical practice.

On the other hand, the GeneXpert assay, offering results in less than $70 \mathrm{~min}$, came closest to the routine SQ-culture methods showing $97.5 \%$ agreement rate. A small discrepancy was due to 2 additional samples positive on the GeneXpert assay that were also positive by the other three methods and therefore included to comprise the extended gold standard panel. GeneXpert thus showed a 100\% match with the extended gold standard panel further utilized in this study. Moreover, while SQ-ETA culture is performed on pus pockets, GeneXpert needed no sample pre-examination and, therefore, was less biased for analysis. We noted that, when taking enrichment-based culture method as reference, GeneXpert showed only $93 \%$ sensitivity. A slightly reduced sensitivity of GeneXpert might be attributable to very low doses of bacteria only detected by enrichment methods, or the presence of substances in the ETA that may interfere with PCR. The latter is supported by the fact that on two occasions (samples 45 and 68, Additional file 1: Table S1) where GeneXpert tests showed errors, a re-analysis with 2-fold sample dilution resolved the issue with high degree of positivity with $\mathrm{Ct}$ of 16 and 33, respectively. GeneXpert is also able to distinguish MRSA and MSSA, and the single sample that was positive for MRSA by SQ-culture results was also positive for the same on the GeneXpert assay. Previous studies showed that GeneXpert assay can be used for detection of $S$. aureus in lower respiratory tract (LRT) samples [13,
14]. In one study, 135 LRT secretions were analysed with the GeneXpert MRSA/SA skin and soft tissue infection (SSTI) assay, which showed a 99\% sensitivity and $72 \%$ specificity with Q-culture as comparator [13]. Another study compared the GeneXpert MRSA nasal assay and qualitative culture for detection of MRSA in transtracheal aspirates and BAL specimens, and showed 93\% concordance between the two assays [14]. The performance of the assays for MRSA detection was not assessed in the latter study [14]. The least specific assay in this study was the in-house nuc gene-based qPCR where $15 \mathrm{~S}$. aureus-positive ETA samples showed no growth on any of the culture methods and were also not detected by the GeneXpert assay. The discrepancies between the quantifications from culture and the molecular methods could be attributed to different factors. For instance, while culture-negative results could arise due to prior antibiotic use or poor sample handling [15], the ability to detect bacteria at low concentrations by qPCR, even from extracellular DNA, could make this test highly non-specific. These findings are in line with previously published studies [16-18]. Interestingly, GeneXpert, also a qPCR method poses no such problem and is most likely due to its capability to detect only whole bacteria through a filter and a washing step that removes extracellular $S$. aureus DNA. Lastly, GeneXpert was the only test that was capable of drastically decreasing the critical time-to-initiation of any potential preventive or therapeutic strategy against VAP, while additionally also allowing detection of MRSA, an important criterion for selecting the right antibiotic.

\section{Conclusion}

S. aureus is a common cause of VAP, a common nosocomial infection associated with a substantial increase in morbidity, mortality as well as in a costly prolongation of patient bed days. With the knowledge that prior colonization with potential pathogens, such as $S$. aureus, plays a significant role in the development of nosocomial infections, rapid and accurate detection of lower respiratory tract colonization and/or infection with $S$. aureus may inform targeted preventive and therapeutic strategies. A recently introduced molecular test, currently utilized for research purposes only, that directly detects $S$. aureus from ETA samples is the GeneXpert SA/MRSA ETA assay. Here, we compared 2 PCR-based (including GeneXpert) and three culture-based methods for $S$. aureus detection in ETAs collected from mechanically-ventilated patients. Although this is a one-centre study on a limited number of samples, we show here for the first time that GeneXpert MRSA/SA ETA is a rapid and sensitive method for $S$. aureus detection in ETA samples. In centres utilizing culture methods, we would recommend increasing assay sensitivity by introducing enrichment-based culture in addition to direct SQ-culture. 


\section{Additional file}

Additional file 1: Table S1. Overview of the results obtained on 79 ETAs using SQ-culture, the GeneXpert assay, Q-culture, enrichment-based culture, and in-house nuc gene-based qPCR. 0-4: negative (0), light (1), moderate (2) and heavy (3). (PDF $88 \mathrm{~kb}$ )

\section{Abbreviations}

$\mathrm{BHI}$ : brain heart infusion; CFU: colony forming units; CNA: colistin-nalidixic acid agar; ETA: endotracheal aspirates; ICU: intensive care unit; LRT: lower respiratory tract; $m e c A$ : methicillin resistance gene; MSSA and

MRSA: methicillin-sensitive and -resistant Staphylococcus aureus; NAC: Nacetylcysteine; nuc: thermostable nuclease; PBS: phosphate buffered saline; Q: quantitative; S. aureus: Staphylococcus aureus; SCCmec: staphylococcal cassette chromosome; spa: staphylococcal protein A; SQ: semi-quantitative; SSTI: skin and soft tissue infection; VAP: ventilator-associated pneumonia

\section{Acknowledgements}

We thank Anouk Vanderstraeten, Gert Leten, Liesbeth Bryssinck and Petra Vertongen (ICU) for excellent technical assistance.

\section{Funding}

The research leading to these results has received support from the Innovative Medicines Initiative Joint Undertaking under grant agreement $\mathrm{n}^{\circ}$ 115523; COMBACTE (Combatting Bacterial Resistance in Europe, resources of which are composed of financial contribution from the European Union's Seventh Framework Programme (FP7/2007-2013) and EFPIA companies' in kind contribution.

JC was supported by the University of Antwerp doctoral assistant funds. LT was supported by COMBACTE.

The sponsors of the study had no role in study design, data collection, data analysis, data interpretation, or writing the report. The corresponding author had full access to all the data in the study and had final responsibility for the decision to submit for publication.

\section{Availability of data and materials}

Please contact the author for data requests.

\section{Authors' contributions}

This study was designed by AR, LY, CL, MM, SK-S, HG, and SMK. Sample collection was done by JC, LT, CL, VM, and PJ. Experimental work was done by $J C, L T$, and LVH. Data were analysed and interpreted by JC, LVH, MI, MM, PJ, SKS, and SMK. Statistical analysis was performed by JC, LY, MI, and SKS. The manuscript was drafted by JC, LVH, MI, SKS, and SMK, and was edited by all authors.

\section{Ethical approval and consent to participate}

All data were retrieved from the patient data management system (Metavision, iMDsoft, Düsseldorf, Germany). The study was reviewed and approved by the hospital's institutional review board (Number 11/2/19 and $16 / 28 / 298$ ) Since all data were fully de-identified, the necessity of obtaining informed consent was waived.

\section{Consent of publication}

Not applicable.

\section{Competing interests}

The authors declare that they have no competing interests.

\section{Publisher's Note}

Springer Nature remains neutral with regard to jurisdictional claims in published maps and institutional affiliations.

\section{Author details}

'Laboratory of Medical Microbiology, Vaccine \& Infectious Disease Institute, University of Antwerp, Universiteitsplein 1, D.S.6.23, 2610 Wilrijk, Belgium. ${ }^{2}$ Medlmmune, Gaithersburg, USA. ${ }^{3}$ Laboratory of Clinical Microbiology, Antwerp University Hospital, Edegem, Belgium. ${ }^{4}$ Department of Critical Care Medicine, Antwerp University Hospital, Edegem, Belgium. ${ }^{5}$ Molecular
Pathology Group, Cell Biology \& Histology, University of Antwerp, Wilrijk, Belgium.

Received: 2 October 2018 Accepted: 21 December 2018

Published online: 05 January 2019

\section{References}

1. Lee AS, de Lencastre H, Garau J, Kluytmans J, Malhotra-Kumar S, Peschel A, et al. Methicillin-resistant Staphylococcus aureus. Nat Rev Dis Prim [Internet]. 2018 May 31;4:18033. Available from: http://www.nature.com/articles/ nrdp201833

2. Chastre J, Fagon J-Y. Ventilator-associated pneumonia. Am J Respir Crit Care Med. 2002:165(23):867-903.

3. American Thoracic Society ; Infectious Diseases Society of America. Guidelines for the management of adults with hospital-acquired, ventilatorassociated, and healthcare-associated pneumonia. Am J Respir Crit Care Med. 2005;15(171):388-416.

4. Jorens PG. Sticking to an old definition of ventilator-associated pneumonia is not old-fashioned. Respir Care [Internet]. 2016;61(3):390-2 Available from: http://rc.rcjournal.com/cgi/doi/10.4187/respcare.04736.

5. Bonten MJ, Weinstein RA. The role of colonization in the pathogenesis of nosocomial infections. Infect Control Hosp Epidemiol [Internet]. 1996;17(3): 193-200. Available from: http://www.ncbi.nlm.nih.gov/pubmed/8708364

6. Paling FP, Wolkewitz M, Bode LGM, Klein Klouwenberg PMC, Ong DSY, Depuydt P, et al. Staphylococcus aureus colonization at ICU admission as a risk factor for developing S.?aureus ICU pneumonia. Clin Microbiol Infect [Internet]. 2017 Jan;23(1):49.e9-49.e14. Available from: http://linkinghub. elsevier.com/retrieve/pii/S1198743X16304281

7. Brusselaers N, Labeau S, Vogelaers D, Blot S. Value of lower respiratory tract surveillance cultures to predict bacterial pathogens in ventilator-associated pneumonia: systematic review and diagnostic test accuracy meta-analysis. Intensive Care Med [Internet]. 2013;39(3):365-375. Available from: http:// www.ncbi.nlm.nih.gov/pubmed/23188467

8. Garcia LS, Isenberg HD. Clinical microbiology procedures handbook; 2010

9. Wolk DM, Struelens MJ, Pancholi P, Davis T, Della-Latta P, Fuller D, et al. Rapid Detection of Staphylococcus aureus and Methicillin-Resistant S. aureus (MRSA) in Wound Specimens and Blood Cultures: Multicenter Preclinical Evaluation of the Cepheid Xpert MRSA/SA Skin and Soft Tissue and Blood Culture Assays. J Clin Microbiol [Internet]. 2009 Mar 1;47(3):823-826. Available from: http://jcm.asm.org/cgi/doi/10.1128/JCM.01884-08

10. Fey A, Eichler S, Flavier S, Christen R, Höfle MG, Guzmán CA. Establishment of a real-time PCR-based approach for accurate quantification of bacterial RNA targets in water, using Salmonella as a model organism Appl Environ Microbiol [Internet]. 2004 Jun;70(6):3618-3623. Available from: http://www. ncbi.nlm.nih.gov/pubmed/15184165

11. Torres A. The new American Thoracic Society/infectious disease Society of North America guidelines for the management of hospital-acquired, ventilator-associated and healthcare-associated pneumonia: a current view and new complementary information. Curr Opin Crit Care [Internet]. 2006; 12(5):444-5 Available from: https://insights.ovid.com/crossref?an=00075198200610000-00013.

12. Eroshenko D, Polyudova T, Korobov V. N-acetylcysteine inhibits growth, adhesion and biofilm formation of gram-positive skin pathogens. Microb Pathog [Internet]. 2017;105:145-52 Available from: https://linkinghub. elsevier.com/retrieve/pii/S0882401017300505.

13. Cercenado E, Marin M, Burillo A, Martin-Rabadan P, Rivera M, Bouza E. Rapid detection of Staphylococcus aureus in lower respiratory tract secretions from patients with suspected ventilator-associated pneumonia: evaluation of the Cepheid Xpert MRSA/SA SSTI assay, J Clin Microbiol [Internet]. 2012:50(12): 4095-7 Available from: http://jcm.asm.org/cgi/doi/10.1128/JCM.02409-12.

14. Oh A-c, Lee jk, Lee Hn, Hong Yj, Chang Yh, Hong S-i, et al. Clinical utility of the Xpert MRSA assay for early detection of methicillin-resistant Staphylococcus aureus. Mol Med Rep [Internet]. 2013 Jan;7(1):11-15. Available from: https:// www.spandidos-publications.com/10.3892/mmr.2012.1121

15. Wolk DM, Picton E, Johnson D, Davis T, Pancholi P, Ginocchio CC, et al. Multicenter Evaluation of the Cepheid Xpert Methicillin-Resistant Staphylococcus aureus (MRSA) Test as a Rapid Screening Method for Detection of MRSA in Nares. J Clin Microbiol [Internet]. 2009 Mar 1;47(3): 758-764. Available from: http://jcm.asm.org/cgi/doi/10.1128/JCM.01714-08

16. Lacroix M, Barraud O, Clavel M, Filiputti D, Prudent S, François B, et al. Rapid quantification of Staphylococcus aureus from endotracheal aspirates of 
ventilated patients: a proof-of-concept study. Diagn Microbiol Infect Dis [Internet]. 2015 Oct;83(2):117-120. Available from: http://linkinghub.elsevier. com/retrieve/pii/S0732889315002175

17. Rios-Licea MM, Bosques FJ, Arroliga AC, Galindo-Galindo JO, Garza-Gonzalez E. Quadruplex real-time quantitative PCR assay for the detection of pathogens related to late-onset ventilator-associated pneumonia. J Microbiol Methods [Internet]. 2010;81(3):232-4 Available from: http:// linkinghub.elsevier.com/retrieve/pii/S0167701210001053.

18. Alonzo TA, Pepe MS. Using a combination of reference tests to assess the accuracy of a new diagnostic test. Stat Med [Internet]. 1999 Nov 30;18(22): 2987-3003. Available from: http://www.ncbi.nlm.nih.gov/pubmed/10544302

Ready to submit your research? Choose BMC and benefit from:

- fast, convenient online submission

- thorough peer review by experienced researchers in your field

- rapid publication on acceptance

- support for research data, including large and complex data types

- gold Open Access which fosters wider collaboration and increased citations

- maximum visibility for your research: over $100 \mathrm{M}$ website views per year

At $\mathrm{BMC}$, research is always in progress.

Learn more biomedcentral.com/submissions 